\title{
Chemical and Physical Changes Induced in Optical Materials under High-Intensity Laser Irradiation
}

\author{
Stavros Pissadakis, ${ }^{1}$ Saulius Juodkazis, ${ }^{2}$ and Jacques Albert ${ }^{3}$ \\ ${ }^{1}$ Institute of Electronic Structure \& Laser (IESL), Foundation for Research and Technology - Hellas (FORTH), \\ P.O. Box 1385, 71110 Heraklion Crete, Greece \\ ${ }^{2}$ Research Institute for Electronic Science, Hokkaido University, North 21 - West 10, CRIS Building, Kita-ku, Sapporo 001-0021, Japan \\ ${ }^{3}$ Department of Electronics, Carleton University, 1125 Colonel By Drive, Ottawa, ON, Canada K1S $5 B 6$
}

Correspondence should be addressed to Stavros Pissadakis, pissas@iesl.forth.gr and Jacques Albert, jacques_albert@carleton.ca

Received 30 October 2008; Accepted 4 November 2008

Copyright () 2008 Stavros Pissadakis et al. This is an open access article distributed under the Creative Commons Attribution License, which permits unrestricted use, distribution, and reproduction in any medium, provided the original work is properly cited.

The laser-driven processes of surface structuring and photosensitivity in optical materials have been widely investigated during the last three decades. These accumulated efforts have promoted the understanding of the underlying physical processes involved and, also, prompted the optimal exploitation of the products of these processes in the photonics and micromanufacturing industry. The scientific research in these fields is mostly focused on the investigation of a fundamental problem "how the physical mechanisms and the products of high-intensity laser irradiation are correlated with specific exposure conditions." In such a basic approach, the high-intensity laser beam plays a twofold role, being simultaneously a precision tool for structuring or modifying the materials and an advanced probe for exploring and monitoring the physical processes occurring in the exposed optical material.

The above two broad scientific interest fields followed a rather parallel and similar evolution trend over the years, exhibiting a strong dependency on the available laser sources, especially their wavelengths and intensities. The $\mathrm{CO}_{2}$ and YAG lasers era occurred in the 1970s and early 1980s, and was succeeded by excimer lasers period which lasted until the beginning of 1990s. This is when the terms "laser micromachining, ablation, and refractive index engineering" were established. However, after then, both fields were revolutionised with the emergence and commercialisation of the femtosecond laser sources, defining a new area that lasts up till today. These laser sources provided unprecedented field intensities of $\mathrm{TW} / \mathrm{cm}_{2}$ magnitude, at pulse durations below a few femtoseconds and at several emission wavelengths. The triggering of "cold," high-order multiphoton interactions opened new horizons in the science and technology of surface structuring and photosensitivity, settling several problems and doubts related to the physics yield and the exploitation potential of these optical material processing approaches. Femtosecond laser technology enabled processes such as the 3D structuring and refractive index engineering of high bandgap optical materials or the complex submicron photopolymerisation manufacturing, manufacturing methods which were considered as exotic only a few years ago.

We believe that launching this special issue is rather timely, since numerous and high impact findings have been presented over the last few years by several research groups. A priority objective of such special interest publication is to bring the communities of photosensitivity and laser processing closer together, thereby strengthening common codes of communication and establishing a parallel scientific vision. The contributions published herein cover a diverse number of research topics and applications in both fields, illustrating both experimental and fundamental aspects of those laser-matter interactions. Among the topics presented are filamentation effects, laser backside etching methods, Bragg grating recording in standard, microstructured optical fibres and thin films using ultrafast laser sources, etching of crystalline materials, photo-polymerisation and basic physics studies in photosensitivity and high intensity material modification. 
Hopefully, this volume will constitute a useful reference guide for the researchers that work in fundamental investigations as well as engineers that utilise laser radiation for developing devices in the sectors of planar and fibre photonics, microoptics, and microfluidics.

Stavros Pissadakis Saulius Juodkazis Jacques Albert 